\title{
Electric Fields Produced by a Charge Density in Ionic Crystals
}

\author{
Herbert S. Bennett \\ Institute for Materials Research, National Bureau of Standards, Washington, D.C. 20234
}

(June 12, 1968)

\begin{abstract}
Spatially extended defects such as the $F$ center give rise to an effective defect charge density which may produce important polarizations in the crystal. The electric field in the crystal depends upon these induced dipoles. Lattice summations for the contribution to the electric field which arises from ionic shells centered about the defect are evaluated for the $\mathrm{NaCl}$ and $\mathrm{CaF}_{2}$ lattice structures.
\end{abstract}

Key Words: $\mathrm{CaF}_{2}$; dipole shell coefficient; electric fields; $\mathrm{NaCl}$; polarization; spatially extended defects.

\section{Introduction}

We shall evaluate in this paper the ionic and electronic polarizations which defects induce in ionic crystals. Such polarizations play a major role in the energy of formation, the mobility, the self energy, and the electronic structure of defects.

We shall consider an ionic crystal which contains one molecule $\mathrm{M}_{n_{+}} \mathrm{X}_{n_{-}}$per volume $v_{c}$ of the crystal. The volume $v_{c}$ is the volume of a unit cell and is given by

$$
v_{c}=\mathbf{a}_{1} \cdot\left(\mathbf{a}_{2} \times \mathbf{a}_{3}\right),
$$

where $\mathbf{a}_{1}, \mathbf{a}_{2}$, and $\mathbf{a}_{3}$ are the primitive translational vectors. For $\mathrm{NaCl}$ and $\mathrm{CaF}_{2}$ structures, the primitive translational vectors are $\mathbf{a}_{1}=(a / 2)(0,1,1)$, $\mathbf{a}_{2}=(a / 2)(1,0,1)$ and $\mathbf{a}_{3}=(a / 2)(1,1,0)$, and the volume of the unit cell is $v_{c}=a^{3} / 4$. The lattice constant $a$ is the cation-cation distance. The nearest neighbor distance (cation-anion) is $r_{0}=(a / 2)$ for $\mathrm{NaCl}$ structures and $r_{0}=(\sqrt{3} / 4) a$ for $\mathrm{CaF}_{2}$ structures. The cations are at

$$
\mathbf{r}^{+}=(a / 2)\left[\left(l_{2}+l_{3}\right) \hat{x}+\left(l_{3}+l_{1}\right) \hat{y}+\left(l_{1}+l_{2}\right) \hat{z}\right] .
$$

The anions for $\mathrm{NaCl}$ structures are at

$$
\mathbf{r}^{-}=\mathbf{r}^{+}+(a / 4)(\hat{x}+\hat{y}+\hat{z}),
$$

and the anions for $\mathrm{CaF}_{2}$ structures are at

$$
\mathbf{r}_{1}^{-}=\mathbf{r}^{+}+(a / 4)(\hat{x}+\hat{y}+\hat{z}),
$$

and at

$$
\mathbf{r}_{2}^{-}=\mathbf{r}^{+}+(a / 4)(-\hat{x}-\hat{y}-\hat{z}) .
$$

The integers $l_{i}$ have the values $0, \pm 1, \pm 2$, etc. The above discussion places a cation at the origin. We shall treat also the case for which an anion is at the origin. For this latter case we have the reference vectors $\mathbf{r}_{a}=(a / 2)(0,0,1)$ for $\mathrm{NaCl}$ structures and $\mathbf{r}_{a}=(a / 4)(-1,-1,-1)$ for $\mathrm{CaF}_{2}$ structures.

We view the anions $X$ and the cations $M$ as polarizable point charges occupying the lattice sites. The anion ionicity is $Z_{a}$ and the cation ionicity is $Z_{c}$. The unit cell is electrically neutral, $n_{+} Z_{c}+n_{-} Z_{a}=0$. We also associate an ionic polarizability $\alpha_{a}^{d}$ or $\alpha_{c}^{d}$ and an electronic polarizability $\alpha_{a}^{e}$ or $\alpha_{c}^{e}$ with each ion in the lattice. These polarizabilities determine the response of the crystal to weak static and high frequency electric fields. We represent an ion which is located at the lattice site $\mathbf{r}_{\nu}$ and which experiences a static electric field $\mathbf{E}\left(\mathbf{r}_{v}\right)$ as a point charge $Z_{a} e$ or $Z_{c} e$ located at $\mathbf{r}_{v}$ and upon which we superpose a point dipole moment

$$
\boldsymbol{\mu}\left(\mathbf{r}_{\nu}\right)=\left(\alpha^{e}+\alpha^{d}\right) \mathbf{E}\left(x_{\nu}\right) .
$$

When the period of the electric field is much shorter than the characteristic period for ionic motion but also longer than the orbital period for the electrons about the ion, the point dipole moment is

$$
\boldsymbol{\mu}\left(\mathbf{r}_{\nu}\right)=\alpha^{e} \mathbf{E}\left(\mathbf{r}_{\nu}\right) .
$$

We assume that the response is linear in the electric 
field. In general, the polarizability is a tensor. However, we choose here for convenience that it be diagonal and isotropic. This is a very reasonable assumption for cubic crystals. When we view an ionic crystal as a dielectric continuum, the static dielectric constant $\epsilon_{0}$ and the high frequency (optical) dielectric constant $\epsilon_{\infty}$ give us information about the ionic and electronic dipoles which are induced by weak electric fields.

For our purposes here, we may represent the defect by a spatially dependent charge density $\rho(r)[1] .^{1}$ For example, the $F$ center in an ionic crystal consists of an electron (the $F$ electron) localized about an anion vacancy. We choose the anion vacancy to be at the lattice site $\mathbf{r}=\mathbf{0}$. We characterize quantum mechanically the $F$ electron by its stationary wave function $\psi_{n}(\mathbf{r})$ with energy eigenvalue $\epsilon_{n}$ where $n$ represents all the quantum numbers of the given state. The $F$ electron gives rise to the spatial charge density

$$
\rho_{r}(\mathbf{r})=-e \psi_{n}^{*}(\mathbf{r}) \psi_{n}(\mathbf{r}) .
$$

Also, we view the anion vacancy as the addition of a positive point charge $Z_{v} e=-Z_{a} e$ at the site $r=0$ $\rho_{v}(\mathbf{r})=Z_{v} e \delta^{3}(\mathbf{r})$. The total charge density $\rho_{d}(\mathbf{r})$ of the $F$ center at $\mathbf{r}=\mathbf{0}$ then becomes.

$$
\rho_{d}(\mathbf{r})=e\left[Z_{v} \delta^{3}(\mathbf{r})-\psi_{n}^{*}(\mathbf{r}) \psi_{n}(\mathbf{r})\right] .
$$

The total electric field which a polarizable ion experiences is the sum of the electric field due to the defect charge density $\rho_{d}(\mathbf{r})$ and the electric field due to the electronic dipoles and the ionic dipoles which the defect charge density induces on all the other ions of the crystal. A. D. Franklin and D. J. Sparks [2] have computed the electric fields produced by a point charge $\rho_{p}(\mathbf{r})=Z_{\text {eff }} e \delta^{3}(\mathbf{r})$ in ionic crystals. We will compute in this paper the electric fields produced by extended charge distributions in the $\mathrm{NaCl}$ and $\mathrm{CaF}_{2}$ lattice structures.

Our intended research on the electronic structure of the $F$ center motivates us to carry out the present computations. The $F$ electron and the lattice, particularly the nearest neighbors to the defect, form a very strongly interacting system which we must treat self-consistently. The polarizations which accompany the $F$ center are most important when we use the polarizable ion theory for the $F$ center [1].

\section{Formulation}

In order to make the present computations feasible, we shall consider only the spherically symmetric part $\rho_{s}(r)$ of the charge density $\rho_{d}(\mathbf{r})$; i.e., we average $\rho_{d}(\mathbf{r})$ over the unit sphere.

$$
\rho_{s}(r)=(4 \pi)^{-1} \int_{0}^{2 \pi} d \phi \int_{0}^{\pi} \sin \phi d \phi \rho(\mathbf{r}),
$$

\footnotetext{
'Figures in brackets indicate the literature references at the end of this paper.
}

where $r=|\mathbf{r}|$. For convenience, we will suppress the subscript $s$.

The electrostatic potential $\phi\left(r_{1}\right)$ at the point $\mathbf{r}_{1}$ due to the spherically symmetric part $\rho(r)$ of the defect charge density $\rho_{d}(\mathbf{r})$ is given by the expression,

$$
\phi\left(r_{1}\right)=\frac{\int_{0}^{r_{1}} \rho(r) r^{2} d r}{r_{1}}+\int_{r_{1}}^{\infty} \frac{\rho(r) r^{2} d r}{r} .
$$

We do not allow the dielectric response of the system to enter in eq (6). The negative gradient of the electrostatic potential then prescribes the electric field $\mathbf{E}_{d}\left(\mathbf{r}_{1}\right)$ at $\mathbf{r}_{1}$ due to the charge density $\rho(r)$;

$\mathbf{E}_{d}\left(\mathbf{r}_{1}\right)=E_{d}\left(\mathbf{r}_{1}\right) \hat{r}_{1}=-\frac{\partial \phi\left(r_{1}\right)}{\partial r_{1}} \hat{r}_{1}=\frac{\int_{0}^{r_{1}} \rho(r) r^{2} d r}{r_{1}^{2}} \hat{r}_{1}$,

where $\hat{r}_{1}=\mathbf{r}_{1} /\left|\mathbf{r}_{1}\right|$ is the unit vector in the direction $\mathbf{r}_{1}$. We also define the average amount of charge $Q(r)$ contained within a sphere of radius $r$ centered about $r=0$,

$$
Q(r)=\int_{0}^{r} \rho\left(r_{1}\right) r_{1}^{2} d r_{1}
$$

Combining eqs (7) and (8) gives us

$$
E_{d}(r)=r^{-2} Q(r) .
$$

The electric field $\mathbf{E}_{d}\left(\mathbf{r}_{\nu}\right)$ at $\mathbf{r}_{\nu}$ due to the defect charge density induces a dipole moment $\boldsymbol{\mu}\left(\mathbf{r}_{\nu}\right)$ on the ion at site $\mathbf{r}_{\nu}$. The electric field at the site $\mathbf{r}_{\nu_{1}}$ due to the dipole moment $\boldsymbol{\mu}\left(\mathbf{r}_{v}\right)$ at the site $\mathbf{r}_{v}$ is

$$
\begin{array}{r}
\mathbf{E}_{\mathrm{dip}}\left(\mathbf{r}_{\nu_{1}} ; \mathbf{r}_{\nu}\right)=\frac{3\left(\mathbf{r}_{\nu_{1}}-\mathbf{r}_{\nu}\right)\left[\boldsymbol{\mu}\left(\mathbf{r}_{\nu}\right) \cdot\left(\mathbf{r}_{\nu_{1}}-\mathbf{r}_{\nu}\right)\right]}{\left|\mathbf{r}_{\nu_{1}}-\mathbf{r}_{\nu}\right|^{5}} \\
-\frac{\boldsymbol{\mu}\left(\mathbf{r}_{\nu}\right)}{\left|\mathbf{r}_{\nu_{1}}-\mathbf{r}_{\nu}\right|^{3}} .
\end{array}
$$

The total electric field which the polarizable ion at $\mathbf{r}_{\nu}$ experiences is the sum of the electric field due to the defect and the electric field due to the dipoles induced on all the other ions except the ion at $\mathbf{r}_{\nu}$,

$$
E_{\mathrm{tot}}\left(\mathbf{r}_{\nu}\right)=\mathbf{E}_{d}\left(\mathbf{r}_{\nu}\right)+\sum_{\nu_{1} \neq \nu}^{\prime} \mathbf{E}_{\mathrm{dip}}\left(\mathbf{r}_{\nu} ; \mathbf{r}_{\nu_{1}}\right)
$$

where the prime denotes a sum over all lattices sites except the site $\mathbf{r}_{\nu_{1}}=\mathbf{r}_{\nu}$. Multiplying the left and right hand sides of eq (11) by the appropriate polarizability for the ion at $\mathbf{r}_{\nu}$ converts eq (11) to a system of $3 N$ equations having $3 N$ unknown variables - the three components of the dipole moments $\boldsymbol{\mu}\left(\mathbf{r}_{\nu}\right)$ at each of the $N$ sites in the lattice. Because $N$ has the same order of magnitude as Avogadro's number, we cannot solve the system of equations given by eq (11). Hence, we must make further approximations. The approxima- 
tions which we will employ to overcome the above formidable task are known as the Mott-Littleton procedure [3].

We divide the crystal into two regions. Region I contains all the ions on shells centered about the defect and having a radius less than or equal to $r_{s}$. The radius $r_{s}$ is the radius of the $s$ th shell of ions centered about the defect. Region II contains all the remaining ions. The Mott-Littleton procedure solves the system of equations given by eq (11) for only region I and employs a continuum model to approximate the solutions for the dipole moments of region II.

We first outline the prescription for the dipole moments in region II. We view region II as a dielectric continuum. The electric field $\mathbf{E}$ and the displacement vector $\mathbf{D}$ are related in region II by the constituitive equation for a dielectric continuum,

$$
\mathbf{D}(\mathbf{r})=\epsilon \mathbf{E}(\mathbf{r})=\mathbf{E}(\mathbf{r})+4 \pi \mathbf{P}(\mathbf{r}),
$$

where $\epsilon$ is the appropriate dielectric constant and the polarization $\mathbf{P}(\mathbf{r})$ is the dipole moment per unit volume,

$$
\mathbf{P}(\mathbf{r})=[(\epsilon-1) / 4 \pi] \mathbf{E}(\mathbf{r}) .
$$

The dipole moment per unit cell at $\mathbf{r}_{l}$ is,

$$
\mathbf{P}_{c}\left(\mathbf{r}_{l}\right)=v_{c} \mathbf{P}(\mathbf{r})=v_{c}[(\epsilon-1) / 4 \pi] \mathbf{E}(\mathbf{r})
$$

The Mott-Littleton presecription asserts that all dipole moments point in a radial direction from the defect and divides the dipole moment per unit cell among the $n_{+}+n_{-}$ions contained in the unit cell in proportion to their individual polarizabilities;

$$
\boldsymbol{\mu}_{\mathrm{II}}\left(\mathbf{r}_{\nu}\right)=\frac{\alpha_{\nu}}{\left(n_{+} \alpha_{c}+n_{-} \alpha_{a}\right.} v_{c} \frac{(\epsilon-1)}{4 \pi} \frac{\mathbf{E}_{d}\left(\mathbf{r}_{\nu}\right)}{\epsilon_{1}} \hat{r}_{v}
$$

When treating the $F$ center problem one must distinguish between the electronic polarizations and the ionic polarizations. The electronic polarizations respond to the rapid changes in $\rho_{d}(\mathbf{r})$ which occur whenever the $F$ electron undergoes a transition from one state to another state. However, the ionic polarizations may not respond to such rapid changes in $\rho_{d}(\mathbf{r})$. Hence, when $\epsilon_{1}=\epsilon_{\infty}$, then $\epsilon$ must be $\epsilon_{\infty}$. But when $\epsilon_{1}=\epsilon_{0}$, $\epsilon=\epsilon_{\infty}$ when we compute only the electronic polarizations and $\epsilon=\epsilon_{0}$ when we compute both the electronic and ionic polarizations. When both ionic and electronic polarizations occur $\alpha_{\nu}=\alpha_{\nu}^{e}+\alpha_{\nu}^{d}$ and when only electronic polarizations occur $\alpha_{\nu}=\alpha_{\nu}^{e}$. Referring to eq (15) we define the polarizability of an ion in region II by the quantity.

$$
M_{\mathrm{II}}(\nu)=\frac{\alpha_{v} v_{c}(\epsilon-1)}{4 \pi \epsilon_{1}\left(n_{+} \alpha_{c}+n_{-} \alpha_{a}\right)} .
$$

The electric field at a site $\mathbf{r}_{1}$ in region I due to all the dipoles [eq (15)] in region II is

$$
\begin{aligned}
\mathbf{E}_{\text {out }}\left(\mathbf{r}_{1}\right)= & \sum_{\substack{\nu \\
r_{\nu}>r_{s}}} \frac{Q\left(r_{\nu}\right) M_{\mathrm{II}}(\nu)}{r_{\nu}^{3}\left|\mathbf{r}_{1}-\mathbf{r}_{\nu}\right|^{3}} \\
& {\left[\frac{3\left(\mathbf{r}_{1}-\mathbf{r}_{\nu}\right)\left\{\mathbf{r}_{\nu} \cdot\left(\mathbf{r}_{1}-\mathbf{r}_{\nu}\right)\right\}}{\left|\mathbf{r}_{1}-\mathbf{r}_{\nu}\right|^{2}}-\mathbf{r}_{\nu}\right] . }
\end{aligned}
$$

The summation is over all the sites in region II. The radial part of $\mathbf{E}_{\text {out }}\left(\mathbf{r}_{1}\right), E_{\text {out }}\left(\mathbf{r}_{1}\right)=\mathbf{E}_{\text {out }}\left(\mathbf{r}_{1}\right) \cdot\left(\mathbf{r}_{1} /\left|\mathbf{r}_{1}\right|\right)$, is given by,

$$
\begin{aligned}
& E_{\text {out }}\left(\mathbf{r}_{1}\right)=\sum_{\substack{\nu \\
r_{\nu}>r_{\nu}}} \frac{Q\left(r_{\nu}\right) M_{\text {II }}(\nu)}{r_{1} r_{\nu}^{3}\left|\mathbf{r}_{1}-\mathbf{r}_{\nu}\right|^{5}} \\
& \times\left[2\left(\mathbf{r}_{\nu} \cdot \mathbf{r}_{1}\right) r_{1}^{2}-3 r_{\nu}^{2} r_{1}^{2}-\left(\mathbf{r}_{\nu} \cdot \mathbf{r}_{1}\right)^{2}+2 \mathbf{r}_{\nu}^{2}\left(\mathbf{r}_{\nu} \cdot \mathbf{r}_{1}\right)\right] .
\end{aligned}
$$

When we express the summation which appears in eq (18) in terms of a summation over the shells, we may evaluate more easily the radial part of $\mathbf{E}_{\text {out }}\left(\mathbf{r}_{1}\right)$. Each shell of ions centered about the defect has ions of the same type and each ion of shell $s$ exhibits the same polarizability $M_{\mathrm{II}}(\nu)=M_{\mathrm{II}}(s)$. Hence, we may write an alternate expression, which is computationally more practical, for the radial field $E_{\text {out }}\left(\mathbf{r}_{1}\right)$; namely,

$$
E_{\text {out }}\left(\mathbf{r}_{1}\right)=r_{0}^{-5} \sum_{s^{\prime}>s} Q\left(\mathbf{r}_{s^{\prime}}\right) M_{\mathrm{II}}\left(s^{\prime}\right) D\left(s^{\prime}, \mathbf{r}_{1}\right) .
$$

The dipole coefficients for each shell $s$, which is outside region $\mathrm{I}, D\left(s ; \mathbf{r}_{1}\right)$ is a summation over the $n(s)$ ions on the $s$ th shell;

$$
\begin{aligned}
& D\left(s ; \mathbf{r}_{1}\right)=\sum_{m=1}^{n(s)} \frac{r_{0}^{5}}{r_{1} r_{s}^{3}\left|\mathbf{r}_{1}-\mathbf{r}_{m}\right|^{5}} \\
& \times\left[2\left(\mathbf{r}_{m} \cdot \mathbf{r}_{1}\right) r_{1}^{2}-3 r_{m}^{2} r_{1}^{2}-\left(\mathbf{r}_{m} \cdot \mathbf{r}_{1}\right)^{2}+2 r_{m}^{2}\left(\mathbf{r}_{m} \cdot \mathbf{r}_{1}\right)\right]
\end{aligned}
$$

where the site $\mathbf{r}_{m}$ is the position of the $m$ th ion of shell $s$ and $\left|\mathbf{r}_{m}\right|=r_{s}$.

We may show from cubic symmetry arguments and from the assumption of radial dipoles that the dipole coefficient $D\left(s, \mathbf{r}_{1}\right)$ evaluated for the lattice site $\mathbf{r}_{1}$ on shell $t$ in region $\mathrm{I}$ is the same for all the other lattice sites $\mathbf{r}_{m}$ on the same shell $t$ in region $\mathrm{I}$.

The system of equations for the dipole moments, eq (11), is tractable only when region I contains a small number of shells; e.g., $u$ shells. Incorporating eqs (19) and (20) into eq (11) and assuming that the dipole moments in region $\mathrm{I}$ also are radially directed, we may reduce the system of equations given by eq (11) to $u$ equations containing the $u$ dipole moments on the ions in region I. The magnitude of each dipole moment at the lattice sites on a given shell will be the same.

\section{Results}

The experience which we have gained from our study of the $F$ center in the alkaline earth oxide $\mathrm{CaO}$ [4] indicates that reasonable agreement with experiment obtains when we use the first order Mott-Littleton pro- 
cedure to evaluate the polarizations associated with the $F$ center. We shall present therefore the first order Mott-Littleton prescription in order to demonstrate a specific example of how one might use the preceding discussion.

The first order Mott-Littleton approximation states that region I contains only the first shell and that region II contains all the remaining shells. The first shell about an anion defect contains $n_{c}$ cations at a distance $r_{1}=r_{0}$ from the defect center. Again, we assume that the induced dipole moment on an ion in the first shell points in a radial direction and has a magnitude $\mu_{1}$. The radial electric field at the site $\mathbf{r}_{1}$ due to the dipole moments $\mu_{1} \hat{r}_{m}$ on the $\left(n_{c}-1\right)$ other ions on the first shell is

$$
E_{1} \hat{r}_{1}=\frac{\mu_{1} C_{1} \hat{r}_{1}}{r_{1}^{3}},
$$

where the same shell dipole coefficient $C_{1}$ is a constant for a given lattice structure. The system of equations represented by eq (11) reduces in the first order MottLittleton approximation to one equation for the one unknown dipole moment $\mu_{1}$; namely,

$$
\mu_{1}=\alpha_{1}\left[\frac{Q\left(r_{1}\right)}{r_{1}^{2}}+E_{\text {out }}\left(r_{1}\right)+\frac{\mu_{1} C_{1}}{r_{1}^{3}}\right] \text {. }
$$

Equation (22) then predicts that the dipole moment $\mu_{1} \hat{r}_{1}$ on the ions in the first shell about the defect is

$$
\mu_{1} \hat{r}_{1}=\frac{\alpha_{1}\left[\frac{Q\left(r_{1}\right)}{r_{1}^{2}}+E_{\text {out }}\left(r_{1}\right)\right]}{\left[1-\frac{C_{1} \alpha_{1}}{r_{1}^{3}}\right]} \hat{r}_{1},
$$

TABLE 1. The dipole coefficients $\mathrm{D}\left(\mathrm{s}, \mathrm{r}_{1}\right)$ for $\mathrm{NaCl}$ structures

The defect site is an anion site and the evaluation site $\mathbf{r}_{1}$ is a cation site on the first nearest neighbor shell centered about the anion defect site. Region I contains only the first nearest neighbor shell. Region II contains all the remaining shells. The number of cations in the first shell is $n_{c}=6$ and the same shell dipole coefficient $C_{1}$ for the $\left(n_{c}-1\right)=5$ other ions on the first shell is $C_{1}=-2.3713$. The radius of shell $s$ is $r_{s}$ in units of $(a / 4)$ and the dipole coefficients $D\left(s, r_{1}\right)$ for the shells outside the first shell are given by eq $(20)$. The lattice constant

\begin{tabular}{|c|c|c|c|c|c|c|}
\hline \multirow{3}{*}{ Shell } & \multirow{3}{*}{$\begin{array}{c}\begin{array}{c}\text { Radius } \\
\text { squared }\end{array} \\
r_{s}^{2}\end{array}$} & \multicolumn{4}{|c|}{ Dipole coefficient $D\left(s, r_{1}\right)$} & \multirow{3}{*}{$\begin{array}{l}\text { Number of ions } \\
\text { in shell }\end{array}$} \\
\hline & & \multicolumn{2}{|c|}{ Cation shell } & \multicolumn{2}{|c|}{ Anion shell } & \\
\hline & & $N$ & $n$ & $N$ & $n$ & \\
\hline $\begin{array}{l}2 \\
3 \\
4 \\
5 \\
6 \\
7 \\
8 \\
9 \\
10 \\
11 \\
12 \\
13 \\
14 \\
15 \\
16 \\
17 \\
18 \\
19 \\
20 \\
21\end{array}$ & $\begin{array}{r}8 \\
12 \\
16 \\
20 \\
24 \\
32 \\
36 \\
40 \\
44 \\
48 \\
52 \\
56 \\
64 \\
68 \\
72 \\
76 \\
80 \\
84 \\
88 \\
96\end{array}$ & $\begin{array}{r}-0.4293 \\
+.4580 \\
-.7591 \\
+.3931 \\
-.1040 \\
+.8232 \\
-.8172 \\
+.1159\end{array}$ & $\begin{array}{l}-0 \\
-1 \\
-2 \\
-2 \\
-2 \\
-3 \\
-3 \\
-3\end{array}$ & $\begin{array}{r}-0.2287 \\
+.3742 \\
-.5176 \\
-.1073 \\
+.1535 \\
-.2678 \\
-.3719 \\
+.1112 \\
+.9372 \\
+.3199 \\
-.6631 \\
-.2046\end{array}$ & $\begin{array}{l}+1 \\
-0 \\
-1 \\
-1 \\
-1 \\
-2 \\
-2 \\
-2 \\
-3 \\
-3 \\
-3 \\
-3\end{array}$ & $\begin{array}{r}12 \\
8 \\
6 \\
24 \\
24 \\
12 \\
30 \\
34 \\
24 \\
24 \\
8 \\
24 \\
48 \\
6 \\
48 \\
36 \\
36 \\
24 \\
24 \\
48 \\
24 \\
24\end{array}$ \\
\hline$\left.r_{1}\right)$ & & -.3881 & & -1.965 & & \\
\hline
\end{tabular}
is $a$ and the nearest neighbor distance is $r_{0}=(a / 2)$. The dipole coefficients are expressed in the form $N \times 10^{\prime \prime}$. where $E_{\text {out }}\left(r_{1}\right)$ is given by eq (19) in terms of the dipole coefficients $D\left(s, \mathbf{r}_{1}\right)$.

We list in tables 1 and 2 the dipole coefficients $D\left(s, \mathbf{r}_{1}\right)$ for the $\mathrm{NaCl}$ and $\mathrm{CaF}_{2}$ structures. The computer program used to evaluate the dipole coefficients may be easily modified to treat defects centered about cations and to treat any number of shells in region I. The results given in tables 1 and 2 are applicable directly to the $F$ center problem.

The author thanks A. D. Franklin for many informative discussions.

TABLE 2. The dipole coefficients $\mathrm{D}\left(\mathrm{s}, \mathrm{r}_{1}\right)$ for $\mathrm{CaF}_{2}$ structures

The defect site is an anion site and the evaluation site $\mathbf{r}_{1}$ is a cation site on the first nearest neighbor shell centered about the anion defect site. Region I contains only the first nearest neighbor shell. Region II contains all the remaining shells. The number of cations in the first shell is $n_{c}=4$ and the same shell dipole coefficient $C_{1}$ for the $\left(n_{c}-1\right)=3$ other ions on the first shell is $C_{1}=-1.1482$. The radius of shell $s$ is $r_{3}$ in units of $(a / 4)$ and the dipole coefficients $D\left(s, r_{1}\right)$ for the shells outside the first shell are given by eq $(20)$. The lattice constant is $a$ and the nearest neighbor distance is $r_{0}=\left(3^{1 / 2} a / 4\right)$. The same shell coefficient $C_{1}$ given

\begin{tabular}{|c|c|c|c|c|c|c|}
\hline \multirow{3}{*}{ Shell } & \multirow{3}{*}{$\begin{array}{c}\begin{array}{c}\text { Radius } \\
\text { squared }\end{array} \\
r_{s}^{2}\end{array}$} & \multicolumn{4}{|c|}{ Dipole coefficient $D\left(s, r_{1}\right)$} & \multirow{3}{*}{$\begin{array}{l}\text { Number of ions } \\
\text { in shell }\end{array}$} \\
\hline & & \multicolumn{2}{|c|}{ Cation shell } & \multicolumn{2}{|c|}{ Anion shell } & \\
\hline & & $N$ & $n$ & $N$ & $n$ & \\
\hline 2 & 4 & \multirow{5}{*}{-.3580} & \multirow{4}{*}{-0} & -0.3170 & +1 & 6 \\
\hline 3 & 8 & & & -.5616 & -0 & 12 \\
\hline 4 & 11 & & & & & 12 \\
\hline 5 & 12 & & & +.3263 & -0 & 8 \\
\hline 6 & 16 & & \multirow{3}{*}{-1} & -.4316 & -1 & 6 \\
\hline 7 & 19 & \multirow[t]{3}{*}{+.8516} & & & & 12 \\
\hline 8 & 20 & & & -.3001 & -1 & 24 \\
\hline 9 & 24 & & & +.1424 & -1 & 24 \\
\hline 10 & 27 & \multirow[t]{2}{*}{-.1084} & -1 & & & 16 \\
\hline 11 & 32 & & & +.5379 & -3 & 12 \\
\hline 12 & 35 & \multirow[t]{2}{*}{-.1090} & \multirow[t]{2}{*}{-1} & & & 24 \\
\hline 13 & 36 & & & +.2722 & -2 & 30 \\
\hline 14 & 40 & & \multirow{3}{*}{-2} & -.3217 & -2 & 24 \\
\hline 15 & 43 & \multirow[t]{3}{*}{+.7107} & & & & 12 \\
\hline 16 & 44 & & & -.8402 & -3 & 24 \\
\hline 17 & 48 & & & +.7626 & -3 & 8 \\
\hline 18 & 51 & \multirow[t]{3}{*}{+.4131} & \multirow[t]{2}{*}{-3} & & & 24 \\
\hline 19 & 52 & & & -.4039 & -4 & 24 \\
\hline 20 & 56 & & & +.7445 & -3 & 48 \\
\hline 21 & 59 & \multirow[t]{2}{*}{-.9259} & \multirow[t]{2}{*}{-3} & & & 36 \\
\hline 22 & 64 & & & -.2120 & -3 & 6 \\
\hline 23 & 67 & \multirow[t]{3}{*}{-.8697} & \multirow[t]{2}{*}{-3} & & & 12 \\
\hline 24 & 68 & & & -.8123 & -4 & 48 \\
\hline 25 & 72 & & & -.2217 & -3 & 36 \\
\hline 26 & 75 & \multirow[t]{3}{*}{-.1190} & \multirow[t]{2}{*}{-3} & & & 28 \\
\hline 27 & 76 & & & +.1574 & -3 & 24 \\
\hline 28 & 80 & & \multirow{3}{*}{-2} & -.8521 & -4 & 24 \\
\hline 29 & 83 & \multirow[t]{3}{*}{+.1069} & & & & 36 \\
\hline 30 & 84 & & & -.3467 & -4 & 48 \\
\hline 31 & 88 & & & +.1588 & -3 & 24 \\
\hline 32 & 91 & \multirow{2}{*}{-.2775} & \multirow[t]{2}{*}{-3} & & & 24 \\
\hline 33 & 96 & & & +.4782 & -4 & 24 \\
\hline & & -.2882 & & -3.464 & & \\
\hline
\end{tabular}
in ref. 2 is expressed in terms of the anion-anion distance and the above value is in terms of the anion-cation distance. The dipole coefficients are expressed in the form $N \times 10^{\prime \prime}$.

\section{References}

[1] Bennett, H. S., Phys. Rev. 169, 729 (1968).

[2] Franklin, A. D., and Sparks, D. J., J. Res. NBS 71A (Phys. and Chem.). No. 1, 1 (1967).

[3] Mott, N. F., and Littleton, M. J., Trans. Far. Soc. 34, 485 (1938).

[4] Bennett, H. S., Bull. Am. Phys. Soc. Ser. II, 13, 420 (1968); and to be published.

(Paper 72A5-514) 